\title{
The Monty Hall Problem and beyond: Digital-Mathematical and Cognitive Analysis in Boole's Algebra, Including an Extension and Generalization to Related Cases
}

\author{
Leo Depuydt \\ Department of Egyptology and Ancient Western Asian Studies, Brown University, Providence, Rhode Island, USA \\ E-mail:Leo_Depuydt@brown.edu \\ Received April 27, 2011; revised May 14, 2011; accepted May 25, 2011
}

\begin{abstract}
The Monty Hall problem has received its fair share of attention in mathematics. Recently, an entire monograph has been devoted to its history. There has been a multiplicity of approaches to the problem. These approaches are not necessarily mutually exclusive. The design of the present paper is to add one more approach by analyzing the mathematical structure of the Monty Hall problem in digital terms. The structure of the problem is described as much as possible in the tradition and the spirit—and as much as possible by means of the algebraic conventions - of George Boole's Investigation of the Laws of Thought (1854), the Magna Charta of the digital age, and of John Venn's Symbolic Logic (second edition, 1894), which is squarely based on Boole's Investigation and elucidates it in many ways. The focus is not only on the digital-mathematical structure itself but also on its relation to the presumed digital nature of cognition as expressed in rational thought and language. The digital approach is outlined in part 1. In part 2, the Monty Hall problem is analyzed digitally. To ensure the generality of the digital approach and demonstrate its reliability and productivity, the Monty Hall problem is extended in parts 3 and 4 to related cases in light of the axioms of probability theory. In the full mapping of the mathematical structure of the Monty Hall problem and any extensions thereof, a digital or non-quantitative skeleton is fleshed out by a quantitative component. The pertinent mathematical Equations are developed and presented and illustrated by means of examples.
\end{abstract}

Keywords: Binary Structure, Boolean Algebra, Boolean Operators, Boole’s Algebra, Brain Science, Cognition, Cognitive Science, Digital Mathematics, Electrical Engineering, Linguistics, Logic, Monty Hall Problem, Neuroscience, Non-quantitative and Quantitative Mathematics, Probability Theory, Rational Thought and Language

\section{Introduction}

The Monty Hall problem, named after the television host Monty Hall who made it famous in a TV show, has received its fair share of attention in mathematics. Recently, accessibility to the history of the problem was greatly enhanced due to the appearance of a monograph devoted entirely to the subject [1]. A multiplicity of approaches has been applied to the problem. These approaches are not necessarily mutually exclusive. Among key contributions of most recent date to the problem's analysis are an updated statement of the Bayesian analysis of the problem [2], a challenge to move towards a mathematical modeling of the problem [3], and yet other innovative treatments [4].

The design of the present paper is to add one more approach by analyzing the mathematical structure of the Monty Hall problem in digital terms. The structure of the problem is described as much as possible in the tradition and the spirit, and by means of the algebraic conventions, of George Boole's Investigation of the Laws of Thought (1854), the Magna Charta of the digital age, and of John Venn's Symbolic Logic (second edition, 1894), which is squarely based on Boole's Investigation and elucidates it in many ways [5].

This paper has four main parts. The digital approach is 
outlined in general in part 1 . In part 2, the Monty Hall problem is analyzed digitally. The Monty Hall problem involves 3 doors, 1 car, 2 goats, 1 picked door, and 1 opened door. To ensure the generality of the digital approach and to demonstrate its reliability and productivity, it would seem to be critically important to extend and generalize the analysis of the Monty Hall problem to any number of doors, cars, opened doors, and picked doors in light of the axioms of probability theory. Such an extension and generalization is the subject of parts 3 and 4 of this paper. Part 3 concerns an extension to any number of doors, cars, opened doors, and picked doors. Part 4 concerns an additional extension to any number of picked doors. The pertinent mathematical Equations are developed and presented and illustrated by means of examples.

\section{Preliminary Considerations and Reflections on Digitality and Cognition}

\subsection{Digital Mathematics and Quantitative Mathematics}

Digital mathematics is the mathematics in which nothing gets bigger or smaller and everything is either On or Off, 1 or 0 . Different notation systems are prevalent in digital mathematics. I prefer the notation used by the Father of the digital age, George Boole.

Digital mathematics needs to be differentiated from the search for the roots of mathematics, a subject to which Bertrand Russell and Kurt Gödel and many others have contributed. These efforts are often called logistics, as opposed to logic. A recent voluminous book on the history of logistics from 1870 to 1940 documents all these contributions in detail. This account also reveals that it seems to be hardly the case that the ultimate roots of mathematics have been once and for all fully uncovered [5].

At the outset of his Elements of Algebra (Vollständige Anleitung zur Algebra), Euler states that mathematics is the science of quantity, the systematic study of that which is capable of increase or diminution. This statement is not fully complete. Digital mathematics is fundamentally different from the mathematics with which one is better familiar.

In digital mathematics, nothing gets bigger or smaller. When one performs a Boolean search on the Internet looking for all that is Paris and in addition all that is Paris-adding Paris to Paris as it were by using the so-called Boolean OR-operator-the information that one gets is not in any way larger than if one had just searched for Paris alone. Adding Paris to Paris does not produce a class or set that is twice as large as Paris. If $p$ is Paris, then $p+p=p$ in Boole's algebra. By contrast, in the familiar mathematics, quantity mathematics, $p+p=2 p$.

Furthermore, when one executes a Boolean search for all entities that have two properties, namely being French and again being French-multiplying French by French as it were by using the so-called Boolean AND-operator-one does not obtain search information that is larger than if one had just searched for all that is French. If $f$ is French, then in Boole's algebra, $f \times f=f$. By contrast, in the more familiar mathematics, quantity mathematics, $f \times f=f^{2}$. Something is getting bigger.

\subsection{Boole's Algebra and the Algebra of Electrical Engineering}

It should be noted that, when Boole's algebra was adopted in electrical engineering, the conventions were switched. Boole's " 0 ” is electrical engineering' $s$ " 1 ” and vice versa. Boole’s “ $\times$ ” is electrical engineering's “+” and vice versa [6]. I assume that Claude Shannon, who adapted Boole's algebra for the design of switching circuits in the 1930s and thus in a sense became the Father of computer science, was the originator of this change. In electrical engineering, 0 is conceived as zero resistance or hindrance and therefore as an open circuit. I personally prefer Boole's notation and will use it in what follows. But as long as it is understood which functions symbols have, it makes no difference whether one or the other notation is used.

\subsection{The Digital Nature of Rational Thought and Language}

The human experience consists entirely of how the brain engages reality outside itself by means of the senses, nothing more, nothing less. This includes any manner in which the brain combines sensory perceptions internally. There are more than the traditional five senses, sight, hearing, smell, taste, and touch. The others include sensing pain, sensing that one is upside down, sensing resistance when pushing, and sensing hunger. Part of the brain's engagement with what is outside itself may be called rational thought and language-as distinct from, say, emotions.

I refrain from defining at this point exactly what is rational thought and what is language in rational thought and language. It is much preferable to begin by regarding the two together as a single large phenomenon. It may in fact be difficult to disentangle the two entirely. After all, to which of the two do any connections between the two belong?

I am personally convinced that rational thought and 
language is entirely digital. This fundamental assumption will serve as a working hypothesis. I have begun constructing a comprehensive model of how rational thought and language proceeds digitally. But the presentation of this model is reserved for another occasion. In seeking inspiration as to what this model might look like in the brain, there is nothing wrong with creating physical templates consisting of magnetic coils and switches or transistors or memristors in an attempt to create a prefiguration of what will be found later in the brain.

The proposed model has nothing to do with any of the many programs now in existence that allow a machine to comprehend, produce, or translate languages. These programs are impressive, as appears from a translation by Google Translate of a passage of Antoine de SaintExupéry's Le petit prince presented in a recent article in the New York Times [7]. These programs do not, however, in any way mimic human language nor do they pretend to. All are based on probability and statistics. Relying on huge databases and much computing power, the programs mathematically predict what is most likely to come next based on information already stored. These intelligent guesses are fast becoming ever more accurate.

\subsection{Empirical Basis for Observing the Digitality of Rational Thought and Language}

Little or nothing is known directly about how the biological brain produces thought and language. The question arises: Is it not premature to construct models pertaining to how the brain thinks and talks? Where is the empirical basis?

The empirical basis is twofold. First, it is abundantly clear that the brain teems with digital activity, even if the precise mechanisms of this activity are mostly not understood. Second, as one brain communicates with another through thought and language, all communications need to travel by air from the mouth of a speaker to the ear of a hearer or by light from the written page to a reader's eyes. There can be no doubt that everything that is essential to the structure of rational thought and language must be conveyed in sound waves or light beams that travel from mouth to ear or from page to eye.

One might object that sounds and written symbols are not the same as operations of neurons inside the brain. Then again, certain operations of neurons generate a structure that is empirically accessible in language. If the structure of the neurons differed from the linguistic structure they spawn, people would say things that differ from what they think that they are saying. Clearly, the structure expressed in language must be exactly the same as the structure formed inside the brain, even if the material platforms that the two inhabit could hardly dif- fer more.

What about the validity of the proposed digital model? It is true that mathematical models have predictive value. Consider the computations relating to a novel kind of bridge construction. The computations are predictive in the sense that, if they are error free, the bridge should not collapse. What is more, the computations are binding. The bridge must be built according to the computations or it will collapse.

The validity of digital mathematics in general has been amply demonstrated by countless applications in telephone circuits and computer science. Still, one cannot conjure up just any fanciful digital analysis of brain operations and simply expect the brain to operate according to it. The digital analysis must meet certain empirical conditions and be comprehensive in the mathematical sense by extending to all possible cases. The digital analysis should be to linguistic reality what mathematics is to physical reality in the field of physics.

\subsection{The Digital Supplements}

When one looks at a page of written text, the 1s and the 0s do not readily jump at the eye. So where is the digital structure? In a course that I might one day teach about the digital nature of rational thought and language, I might begin by confronting students with the expression "two black cats" and ask where the mathematics is in this expression. I would suspect that quite a few might point to "two" as the mathematical component. However, "black cats" is just as mathematical.

In digital terms, the presence of something creates a certain awareness of its absence, in other words, of all that it is not. Accordingly, to the class or set of cats corresponds a supplement class or supplement of all that is not cats. If the class of cats is denoted by $c$, then its supplement is denoted in Boole's notation by $1-c$, that is, the universe or all that one could possibly think about (Boole's " 1 ") minus (-) cats (c), or also by $\bar{c}$. Likewise, the class of all that is black can be denoted by $b$ and its supplement, all that is not black, by $\bar{b}$.

\subsection{The Digital Combination Classes}

Furthermore, digitally speaking, two classes "black" (b) and "cats" $(c)$, along with their respective supplements, divide the universe or all that one could possibly think about into exactly four combination classes, black cats, non-black cats, what is black but not a cat, and what is neither black nor a cat. The present writer belongs to the fourth category. In Boole's notation, the combination classes are denoted by $b \times c$ (or $b c$ ), $b \times \bar{c}$ (or $b \bar{c}$ ), $\bar{b} \times c$ (or $\bar{b} c$ ), and $\bar{b} \times \bar{c}$ (or $\bar{b} \bar{c}$ ). The universe or all 
that one could possibly think about, Boole's “ 1 ,” consists entirely of the sum of the four combination classes, as follows.

$$
\begin{aligned}
& 1=b \times c+b \times \bar{c}+\bar{b} \times c+\bar{b} \times \bar{c} \\
& \text { (or also }: 1=b c+b \bar{c}+\bar{b} c+\bar{b} \bar{c} \text { ) }
\end{aligned}
$$

This division is profoundly digital. It encompasses all the combinations in which presence and absence, or On and Off, or 1 and 0, of two classes "cat" and "black" and their supplements can combine.

The division is also fundamental to how we think. Consider the simple sentence "The cat is black." The two classes "the cat" and what is black generate four combination classes. What matters is the abolition of one of the four combination classes, namely what is both the cat and not black. It is this operation of abolition that makes the thought "The cat is black" possible.

In Boole's algebra, there are two levels of thought, the primary level of the things and the secondary level of the events. A proposition such as "The sun shines" is primary. The secondary level can be comprised of two primary propositions. An example is "When the sun shines, I take a walk on the beach.” In this sentence, one digital combination class is switched off or shut down or empty, that is, all the occasions when the sun shines and I do not take a walk on the beach. There is no such thing according to said statement. The three other digital combination classes are on or occupied: either the sun shines and I walk on the beach, or the sun does not shine and I walk on the beach, or the sun does not shine and I do not walk on the beach.

The relation to the conditio sine qua non can be explained digitally [8]. Compare the statement already mentioned, "When the sun shines, I take a walk on the beach," with the statement "Only when the sun shines do I take a walk on the beach." A different digital combination class is switched off in the latter statement, namely all the occasions when the sun does not shine and I do take a walk on the beach.

Furthermore, in the sentence "I take a walk on the beach if and only if the sun shines," both said combination classes are switched off. Since there are four digital combination classes, that means that two combination classes remain switched on: either I walk on the beach and the sun shines or I do not walk on the beach and the sun does not shine.

\subsection{Limits to the Universe}

When two classes and their supplements partition the universe or all that one could possibly think about into four combination classes, it is common to impose un- spoken or explicit limits on what is being partitioned [9]. One hardly always considers everything thinkable. For example, the statement "Manchester is the winner" describing the outcome of a soccer match between Manchester United and Liverpool involves four digital combination classes: all that is Manchester and the winner, all that is Manchester but not the winner, all that is not Manchester yet the winner, and all that is neither Manchester nor the winner. The design of the statement "Manchester is the winner" is to present two combination classes as empty: all that is Manchester but not the winner and all that is not Manchester yet the winner. It seems clear that all that is not Manchester does not in this case include the Queen of England or the Pope in Rome. Non-Manchester is limited to the soccer club Liverpool. It is also clear that "Manchester" does not refer to all of the city of Manchester, but just to the soccer club Manchester United. Although the limits imposed on the universe are not stated explicitly, it seems clear what they are.

\subsection{Excursus: On Negation and on the Digitality in Rational Thought and Language}

In a digital world, negation is the mother of all meanings. Everything without exception can be negated: "Caesar" as "not Caesar," "It rains” as "It does not rain," "there” as "not there," “There is" as “There ain't," and "yes” as "no." It is as if reality presents itself to us in two parallel universes, the affirmative and the negated. But what is negation?

E. Schröder, the mathematician and onetime director of the Technische Hochschule in Karlsruhe, advised great caution when it comes to defining negation because the most famous philosophers from Aristotle to Kant proposed definitions of negation that are very far apart and great authorities constructed untenable theories about negation that exhibit the greatest internal contradictions [10]. Greek philosophers struggled mightily with being and not being and being and becoming and the like. But it is only since the mid-nineteenth century that digital mathematics has provided what I believe to be the valid and final definition of negation. What is negation in digital terms?

As the brain engages reality outside itself as observed or as remembered or even as recombined, it naturally does not focus on, or contemplate, everything all at once. It selects certain components of what Boole calls the universe, that is, all that one could possibly think about. To a certain degree, by the way it is structured, reality presumably more easily draws the attention of the brain to certain of its facets rather than to others. One may 
focus on an entity such as a tree, on a property that comes with that entity such as green, or on a circumstance in which that entity along with its property finds itself such as "in the forest." The contemplation of any component of all that one could possibly think about automatically conjures up the notion of all that remains, all that that component is not, for example, all that is not a tree, all that is not green, or all that is not in the forest.

If the component of the universe of thought is viewed as a class or set, then all that is not that component is just as much a class. A class that encompasses all that something is not may be called a supplement class or a supplement. As was noted above, in Boole's algebra, if lower case $g$ denotes all that is green, then all that is not green is denoted by $1-g$, that is, the universe (Boole's " 1 ") minus $g$, which Boole abbreviates as $\bar{g}$.

Negation is born, and so is digitality, when the need arises to refer explicitly to all that something is not. For example, I may want to state that a certain car falls outside the class of all that is green. I can do so by means of the word "not," as in "This car is not green."

Importantly, a class and its supplement together make up all that is thinkable. In this connection, Aristotle formulated the fundamental axiom of thought, namely that something cannot at the same time be and not be something. What is more, the relation between a class and its supplement is like a toggle. The original class is of course all that its supplement class is not. Put differently, the original class is the supplement of its own supplement. One common way of referring to this property of digital reality is that two negations cancel one another.

This is not the place to illustrate the digital component of rational thought and language at length. Suffice it to point to a semantic field among whose members are English words such as "alone," "also," "only," "other," and "self" and an English expression such as "for his part" along with their equivalents in other languages [11]. Everyone knows how to use these words. But defining them is another matter. Without entering into detail, it would appear that these words all refer to digital supplement classes. For example, "he alone" means "no others besides him," "no non-he's" as it were. "He also" means "others besides him," that is, "non-he's" in addition to him. In a digital world, there is also a need for referring with an explicit word to the supplement class. The word "other" performs exactly that function. "Other" refers to what something else that has been mentioned is not.

In this connection, I have also proposed to analyze contrastive emphasis digitally [12]. When one says, for example, "It is in Paris that the session is held" or "The session is held in Paris," one apparently means "not somewhere else," that is, "not in non-Paris." Paris is presented as the digital supplement of its digital supplement, which is very much Paris itself.

\section{Two Goats and a Car: Digital-Mathematical Analysis of the Monty Hall Problem}

\subsection{Boole and Probability}

It is now generally forgotten that Boole wrote the Magna Charta of the digital age, his Investigation of the Laws of Thought (1854), to address problems in probability. But his contribution to probability has been "simply bypassed by the history of the subject" [13]. Boole believed that the theory of probability is a field of mathematics straddling the fence that separates quantitative mathematics from digital mathematics.

It is assumed here as a working hypothesis that the digital approach permeates all engagement of the brain with reality, and that includes assessments of probability. In support of this assumption, the Monty Hall problem is analyzed digitally in the present part 2 and then extended and generalized in light of the axioms of probability theory in parts 3 and 4.

The study of the Monty Hall problem has a long history. But the countless technical and popular treatments of the problem are characterized by the exclusion of a potentially fertile additional approach, the digital and Boolean perspective, the perspective that I personally believe reflects the fundamentally digital nature of how the brain processes reality in terms of rational thought and language.

\subsection{Description of the Monty Hall Problem}

Behind 3 closed doors, 2 goats and 1 car are hiding. One is asked to pick a door to get what is behind it. The aim is to get the car. One begins by picking a closed door without however knowing or being told what it is hiding. Subsequently, someone who knows what is behind all 3 doors without revealing this knowledge to the person who has picked a closed door opens 1 of the 2 doors that were not picked, and more specifically a door hiding a goat. Since 2 of the 3 doors hide goats, it is always possible to open a non-picked door that hides a goat. The other 2 doors remain closed and 1 of these 2 is the one that was initially picked.

The Monty Hall problem revolves entirely around the following question. Once a door has been opened to reveal a goat, should one switch from the closed door that one has picked to the other door that remains closed in 
order to improve one's chances of getting the car? There is no doubt whatsoever that one should switch. The need is therefore for establishing the respective probabilities of switching and not switching doors.

\subsection{Intuitive Grasp of the Need to Switch Doors: Opening Doors to Reveal Goats as a Means of Compressing or Condensing a Probability into Fewer Doors}

The need for switching doors to improve one's chances of getting the car is obvious from the following consideration. Everyone knows that one has only 1 chance in 3 of getting the car by picking a certain door. That in effect means that there are 2 chances in 3 that the car is hiding behind 1 of the 2 other doors that were not picked. In other words, there are 2 chances in 3 that the 2 non-picked doors hide 1 car and 1 goat. Accordingly, there ought to be every temptation to switch to the other 2 doors to get the car. The problem is that one cannot switch to both of the 2 other doors that were initially not picked. One can only switch to 1 of them. But to which one of the 2 should one switch?

This is where critical and odds-changing help arrives in the form of someone opening 1 door to reveal 1 goat. The effect of this intervention is that the chance of 2 in 3 that the 2 other doors are hiding the car is concentrated in 1 unopened door. What opening 1 door to reveal 1 goat achieves is to compress or condense a certain degree of probability distributed equally over a number of doors into fewer doors. In the case of the Monty Hall problem, a probability of $2 / 3$, which is distributed equally as a probability of $1 / 3$ over each of the 2 doors that are initially not picked, is compressed into 1 door by opening 1 door to reveal 1 goat.

In sum, there is every reason to switch doors. It is not certain that one will get the car. But one has 2 chances in 3 of being lucky.

\subsection{The Two Digital Levels, the Level of Things and the Level of Events}

Just as two classes "black" and "cat" digitally generate four combination classes (§2.6), the Monty Hall problem in a digital and Boolean perspective fundamentally involves two classes generating four combination classes. But in the case of the Monty Hall problem, the two classes do not contain all the instances of two things, such as "black things" and "cats," but rather all occasions or instances or occurrences of two events. According to Boole's analysis, rational thought and language exhibits two levels, the level of primary propositions that is concerned with classes of things and the level of sec- ondary propositions that is concerned with classes of events. Each class of events contains all the occurrences of a certain event.

When two classes of events and their supplement classes are partitioned into four combination classes, it is common to impose unspoken or explicit limits on what is being partitioned (§2.7). The same applies to the Monty Hall problem and any extensions thereof. What one is considering in terms of things is limited to a number of doors hiding either cars or goats. What one is considering in terms of events is limited to initially picking or not picking a car and then picking or not picking a car by switching after one or more doors have been opened to reveal goats.

\subsection{Mathematical Notation of Things and Events}

To differentiate things and events in mathematical notation, things are denoted below by lower case italic letters and events by upper case italics. For example, $c$ stands for "cars" and $C$ for picking a door hiding a car. In addition, subscript letters provide additional distinctive information about events. For example, $C_{i}$ will stand for initially picking a door hiding a car.

\subsection{The Two (Classes of) Events Involved in the Monty Hall Problem, the Second Dependent on the First}

In the case of the Monty Hall Problem, two events are involved. What is more, the two events occur in a fixed sequence. The second event always follows the first and is dependent upon the first according to the definition of dependence in the classic theory of probability.

The two classes of events are as follows. The first contains all the occasions when one initially picks the door behind which the car is hiding. The second contains all the occasions when one picks the door behind which the car is hiding by switching from the door initially picked to the sole door that remains closed after a door has been opened to reveal a goat.

\subsection{The Supplement Classes, or Supplements, of the Two Events Involved}

Digitally speaking, all classes come with supplement classes or supplements. A supplement contains all that a class is not. A supplement is a class in its own right. On the level of things, the supplement of "cat" is all that is not a cat. On the level of events, the supplement of "It rains" is all the occasions when it does not rain.

In the case of the Monty Hall problem, the supplement 
of initially picking the door hiding the car contains all the occasions when one fails to initially pick that door. The supplement of picking the door hiding the car by switching from the door initially picked to the sole other door remaining closed after a door has been opened to reveal a goat contains all the occasions when one fails to pick the door hiding the car by switching doors.

\subsection{The Two Classes and Their Supplements in Boole's Algebra}

In Boole's algebra, picking the door hiding the car may be denoted by $C$ and failing to pick that door by $\bar{C}$. Boole uses overstrike as an abbreviation of $1-$, as in $1-C$, that is, the universe or any events that one could possibly think about minus (-) all the occasions when the door hiding the car is picked.

Initially ( $i$ ) picking the door hiding the car may be denoted by $C_{i}$ and failing to initially pick that door by $\bar{C}_{i}$. Furthermore, picking the door hiding the car by switching ( $s$ ) doors once a door has been opened to reveal a goat may be denoted by $C_{s}$ and failing to pick that door by switching doors by $\bar{C}_{s}$.

Since initially picking a car $\left(C_{i}\right)$ is the same as initially not picking a goat $\left(\bar{G}_{i}\right)$, and so on, the following Equations apply.

$$
C_{i}=\bar{G}_{i} \quad \bar{C}_{i}=G_{i} \quad C_{s}=\bar{G}_{s} \quad \bar{C}_{s}=G_{s}
$$

\subsection{The Four Digital Combination (Classes of) Events}

In digital fashion, two classes of events along with their supplements generate four combination classes corresponding to all the four possible combinations of the occurrences and non-occurrences of the two events. The four combination events are as follows:

(1) (On-On) initially pick the car and then again pick the car by switching doors;

(2) (On-Off) initially pick the car but then fail to pick the car by switching doors;

(3) (Off-On) initially fail to pick the car but then succeed in picking the car by switching doors; and

(4) (Off-Off) initially fail to pick the car and then again fail to pick the car by switching doors.

Digital combination class (1) is denoted in Boole's notation by $C_{i} \times C_{s}$ or as $C_{i} C_{s} ; C_{i} \cdot C_{s}$ is yet another notation. Boole's “ $x$ ”- -now better known as the Boolean AND-operator even if Boole himself did not refer to it in this-manner denotes a combined event in which two component events are valid at the same time. The other three combination events may be denoted by $C_{i} \bar{C}_{s}, \bar{C}_{i} C_{s}$, and $\bar{C}_{i} \bar{C}_{s}$.

\subsection{The Probabilities of the Digital Combination Events}

In Boole's algebra, four symbols such as $C_{i}, \bar{C}_{i}, C_{s}$, and $\bar{C}_{s}$ not only denote classes of events but also the probability that the events in question will take place. Accordingly, the probabilities of the four digital combination events (1), (2), (3), and (4) listed in \$3.9 consist for each combination event of the product of the probability that the first of the two combined events will take place multiplied by the probability that the second event will. The four probabilities may therefore be denoted by $C_{i} \times C_{s}, C_{i} \times \bar{C}_{s}, \bar{C}_{i} \times C_{s}$, and $\bar{C}_{i} \times \bar{C}_{s}$ respectively. The symbol " $x$ " is then to be understood quantitatively and not as an equivalent of what is now known as the Boolean AND-operator.

\subsection{1. $100 \%$ as the Sum of the Probabilities of All Combination Events}

The two classes “cat” and "anything black" along with their supplements subdivide all that is thinkable in terms of things, as denoted by Equation (1) in §2.6. Likewise, the two classes $C_{i}$ and $C_{s}$, along with their supplements subdivide all that is thinkable in terms of events, as denoted by Equation (2).

$$
1=C_{i} \times C_{s}+C_{i} \times \bar{C}_{s}+\bar{C}_{i} \times C_{s}+\bar{C}_{i} \times \bar{C}_{s}
$$

In other words, the totality of all possible scenarios consists entirely of four combination events: either $C_{i}$ and $C_{s}$ both happen, or $C_{i}$ does and $C_{s}$ does not, or $C_{i}$ does not but $C_{s}$ does, or neither $C_{i}$ nor $C_{s}$ do. Put differently, either $C_{i}$ and $C_{s}$ co-occur, or $C_{i}$ and $\bar{C}_{s}$ do, or $\bar{C}_{i}$ and $C_{s}$ do, or $\bar{C}_{i}$ and $\bar{C}_{s}$ do. There are no other possibilities.

Accordingly, the probabilities of the four combination events add up to 1 or $100 \%$. It is one hundred percent certain that one of the four combination events will take place.

\subsection{The Two Empty Digital Combination Classes of Events}

Closer reflection reveals that two of the four combination events involved in the Monty Hall problem never occur, namely $C_{i} \times C_{s}$ and $\bar{C}_{i} \times \bar{C}_{s}$. The corresponding classes of events are therefore empty. Digitally speaking, these two combination events are switched off, as it were. 
As regards $C_{i} \times C_{s}$, one cannot initially pick the car and then again pick the car by switching doors because there is only 1 car. As regards $\bar{C}_{i} \times \bar{C}_{s}$, one cannot initially fail to pick the car-in other words, pick a goatand then again fail to pick the car-that is, again pick a goat-by switching doors. The reason is that, when one has initially picked a goat, the door hiding the only other goat is opened and one cannot switch to that door because it is now open. Accordingly, the only door to which one can switch hides the car and one cannot fail to pick the car by switching doors.

The probability of these two combination events is therefore equal to zero (0). Equation (2) in \$3.11, which denotes the sum of the probabilities of the four digital combination events, can therefore be reduced to a sum of two combination events, as follows.

$$
1=C_{i} \times \bar{C}_{s}+\bar{C}_{\mathrm{i}} \times C_{s}
$$

\subsection{The Digital Combination Event Including the Desired Outcome of Getting the Car $(C)$, namely $\bar{C}_{i} \times C_{s}$}

The desired outcome of the Monty Hall problem as a challenge is getting the car $(C)$. Of the two digital combination classes of events that are not empty in Equation (2) in §3.12, namely $C_{i} \times \bar{C}_{s}$ and $\bar{C}_{i} \times C_{s}$, only $\bar{C}_{i} \times C_{s}$ includes the desired outcome $C$. Importantly, this combination event involves switching doors, as denoted by subscript $s$, after initially failing to pick the car $\left(\bar{C}_{i}\right)$. In the other combination event, one had initially picked the car $C_{i}$, but then loses it by switching doors.

If the probability of the combination event with the desired outcome is lower than 0.5 or $50 \%$, one should not switch doors to increase one's chances of getting the car. If the probability is higher than 0.5 or $50 \%$, switching doors makes getting the car more probable. If the probability is exactly 0.5 or $50 \%$, it does not make a difference whether one switches doors or not; one does not increase or decrease one's chances of getting the car. What is the probability of the combination event that includes the desired outcome?

Two of the four digital combination classes of events are empty (\$3.12). Accordingly, the sum of the probabilities of the two other combination events is 1 or $100 \%$. Either one or the other of the two other combination events must be the case.

If the probability of one of the two combination events is $x \%$, then the probability of the other is $(1-x) \%$ because there are only two. The probability of one combination event can be derived from the probability of the other by subtracting the probability of the other from 1 or $100 \%$.

\subsection{The Probability of the Digital Combination Event Including the Desired Outcome, namely $\bar{C}_{i} \times C_{s}$}

The desired outcome is achieved by switching doors as part of the digital combination event $\bar{C}_{i} \times C_{s}$. In order to compute the probability of this combination event, it is first necessary to establish the probabilities of its two components, the two events $\bar{C}_{i}$ and $C_{s}$.

The probability of $\bar{C}_{i}$, that is, failing to initially pick the car, is evidently $2 / 3$ or about $66.7 \%$. There are 3 doors and 2 of them hide a goat.

In establishing the probability of $\bar{C}_{s}$, it needs to be taken into account that $\bar{C}_{s}$ is a dependent event according to the definition of dependence in classical probability theory. Accordingly, the question to ask is: What is the probability that one will pick the car by switching $\left(C_{s}\right)$ after having initially failed to pick it $\left(\bar{C}_{i}\right)$ ? That probability is 1 or $100 \%$. Indeed, if one first picked a goat and the other door hiding a goat is opened, switching to the only other unopened door must always result in picking the car.

The probability of the desired combination event $\bar{C}_{i}$ $\times C_{s}$, which results in picking the car by switching doors $\left(C_{s}\right)$, is therefore $2 / 3 \times 1=2 / 3$. Not only does this combination event produce the desired outcome but its probability is also higher than 0.5 or $50 \%$. There can be no doubt: One has to switch doors to improve one's chances of getting the car.

\subsection{The Probability of the Combination Event That Fails to Achieve the Desired Outcome, namely $C_{i} \times \bar{C}_{s}$}

Failing to get the car is the outcome of the digital combination event $C_{i} \times \bar{C}_{s}$. After initially picking the car $\left(C_{i}\right)$, one loses it by switching doors $\left(\bar{C}_{s}\right)$. The probability of this digital combination event can be derived directly from the probability of the other digital combination event $\bar{C}_{i} \times C_{s}(\S 3.14)$. That is because only two of four possible digital combination events actually occur in the Monty Hall problem. Their combined probability must therefore be 1 or $100 \%$. Either one or the other must take place. Since the probability of $\bar{C}_{i} \times C_{s}$ is $2 / 3$ ( $\S 3.14)$, the probability of $C_{i} \times \bar{C}_{s}$ must be $1-2 / 3$ or $1 / 3$. But for completeness' sake, it may be desirable to establish the probability of $C_{i} \times \bar{C}_{s}$ in its own right.

The probability of initially picking the car $\left(C_{i}\right)$ is $1 / 3$ or about $33.3 \%$. There is 1 car and there are 3 doors. But once the car is chosen, the probability of failing to pick the car by switching doors $\left(\bar{C}_{s}\right)$ is 1 or $100 \%$ because one has already picked the sole car and cannot pick it 
again. Consequently, the probability of $C_{i} \times \bar{C}_{s}$ is $1 / 3 \times 1$ or $1 / 3$.

\section{Extension and Generalization of the Monty Hall Problem to Any Number of Doors (d), Cars (c), and Opened Doors (o)}

\subsection{The Probability of Getting a Car by

$$
\text { Switching Doors }\left(C_{s}\right): \frac{c(d-1)}{d(d-1-o)}
$$

The general question that stands at the center of the larger problem of which the Monty Hall problem represents just one specific case is as follows: Should one switch doors in order to improve one's chances of getting cars after doors have been opened to reveal goats? To answer the question, one needs to know the respective probabilities of picking a door hiding a car by switching doors $\left(C_{s}\right)$ and picking a door hiding a car by not switching. The probability of picking a door hiding a car by not switching is the same as the probability of initially picking a door hiding a $\operatorname{car}\left(C_{i}\right)$. If one does nothing else, the probability does not change. If the probability of getting a car by switching is greater than the probability of getting a car by not switching, then one should switch doors to improve one's chances of getting a car. A comparison between the probabilities of $C_{s}$ and $C_{i}$ imposes itself.

Of the two probabilities in question, that of picking a door hiding a car by not switching is readily known. As was said, it is the same as the probability of initially picking a door hiding a car $\left(C_{i}\right)$. This probability is the ratio of cars to doors and can be denoted by $c / d$. It is the ratio of favorable outcomes to the sum of favorable and unfavorable outcomes, in accordance with a fundamental axiom of classical probability theory. For example, if there is $1 \mathrm{car}$ and there are 3 doors, the chance of initially picking a door hiding a car is $1 / 3$ and $1 / 3$ remains the chance of getting the car if one does nothing else, such as switching to another door. If there are 2 cars and 5 doors, the chance of getting a car by sticking with the door that was initially picked is $2 / 5$. And so on.

As regards the other probability, that of getting a car by switching doors $\left(C_{s}\right)$, Equation (4) applies.

$$
C_{s}=\frac{c(d-1)}{d(d-1-o)}
$$

$c=$ the number of cars ( $=\bar{g}$, the number of non-goats)

$d=$ the number of doors $(=c+g$, the sum of cars and goats)

$o=$ the number of opened doors

How Equation (4) is obtained is explained below in
$\S \S 4.4-6$. In Equation (4), 1 is the number of picked doors $(p)$. The picked doors are not denoted algebraically by, say, $p$ because Equation (4) is only valid when there is only 1 picked door, that is, when $p=1$. Cases in which there is more than 1 picked door, that is, in which $p>1$, are discussed in part 5 below.

Equation (4) does not feature goats $(g)$. However, of the three variables $d, c$, and $g$, each can be derived from the two others because $d=c+g, c=d-g$, and $g=d-c$.

When the number of the cars is 1, Equation (4) can be simplified as follows.

$$
C_{s}=\frac{c g}{d(g-o)}=\frac{g}{d(g-o)}
$$

After all, (4) is equivalent to the following Equation.

$$
C_{s}=\frac{c(c+g-1)}{d(c+g-1-o)}
$$

If $c=1$, then the following Equation can be substituted for (6).

$$
C_{s}=\frac{1(1+g-1)}{d(1+g-1-o)}
$$

And Equation (7) can be rewritten as Equation (5). As will be seen below, the deeper reason for this simplification is as follows. When there is more than 1 car, getting a car by switching doors can be the result of both initially picking a door hiding a car and initially picking a door hiding a goat. There are two scenarios. When there is only 1 car, getting the car by switching doors can only be the result of initially picking a door hiding a goat. There is only one scenario. One cannot initially pick the only car and then pick it again when switching doors.

\section{2. $(d-1) /(d-1-o)$ as the Factor by Which}

\section{the Probability of Getting a Car by Not Switching Doors Increases by Switching Doors After Doors Have Been Opened to Reveal Goats}

Equation (4) can also be rewritten as follows.

$$
C_{s}=\frac{c}{d} \times \frac{d-1}{d-1-o}
$$

The first member of the right-hand term in Equation (8), $c / d$, is the probability of getting a car by not switching. As was noted earlier, this probability is the same as the probability of initially picking a door hiding 
a car $\left(C_{i}\right)$. The probability $C_{i}$ cannot change if one does nothing else. Equation (8) can therefore also be written as follows.

$$
C_{s}=C_{i} \times \frac{d-1}{d-1-o}
$$

The second member of the right-hand term in Equation (9), $(d-1) /(d-1-o)$, is the factor by which the probability of getting a car by not switching doors is changed by actually switching.

When there is only 1 car, then Equation (8) can be written in light of Equation (5) in $§ 4.1$ as follows.

$$
C_{s}=\frac{C}{d} \times \frac{g}{g-o}
$$

The factor by which the probability of getting a car by not switching doors is changed by actually switching is then $g /(g-o)$.

If no doors are opened to reveal goats, then $o=0$ and the factor in question is $(d-1) /(d-1)$ (or $g / g$ when there is only $1 \mathrm{car}$ ), that is, 1 . In this specific case, Equation (9) is equivalent to the following Equation.

$$
C_{s}=C_{i} \times 1=C_{i}
$$

The probability does not change by switching doors and one hence does not increase one's chances of getting the car by switching.

If one or more doors are opened, then $o>0$ and $d-1-o$ is smaller than $d-1$. It follows that $(d-1) /(d-1-o)$ is larger than 1 when any doors are opened. In this case, Equation (9) is equivalent to the following Equation.

$$
C_{s}>C_{i}
$$

In sum, one will always improve one's chances of getting a car if one switches doors after doors have been opened to reveal goats. The increase in the probability of getting the car will vary. For example, it will be small if there are many, many doors and only a couple of doors hiding goats are opened. But there will always be an increase.

What is more, any increase in the number of opened doors $(o)$ produces an increase in the probability of getting the car by switching doors $\left(C_{s}\right)$. As $o$ in $(d-1) /(d-1-o)$ increases, $d-1-o$ decreases. Accordingly, the fraction as a whole increases and so does $C_{s}$.

Any increase in the number of doors hiding cars $(c)$ increases the chances of getting a car in general and any increase in the number of doors hiding goats $(g)$ decreases those chances in general. But any changes in the number of cars or doors do not affect the probability of getting a car by switching doors. Only the number of opened doors $(o)$ does.

\subsection{Examples}

\subsubsection{Opening Doors to Reveal Goats Always Increases the Probability of Getting a Car by Switching Doors}

Equation (4) in §4.1 defines the probability that one will get a car by switching doors and makes it possible to compare this probability with the probability of getting a car by not switching. It is a fact that the probability of getting a car will always remain the same when no doors are opened to reveal goats and will always increase when one or more doors are opened. There is therefore never any point in switching doors to get a car when no doors are opened to reveal goats and always reason to switch when doors are opened.

The factor by which the probability in question increases by switching doors when doors are opened is $(d-1) /(d-1-o)$ in general and $g /(g-o)$ in the special case in which there is only $1 \mathrm{car}(\S 4.2)$.

\subsubsection{The Special Status of the Monty Hall Problem}

The Monty Hall problem is just one instance of a much more general problem. The Monty Hall problem is special in that it presents what may be called the minimal scenario of the general problem. It exhibits the lowest numbers of doors, cars, and opened doors. The number of cars cannot be lowered because there is only one. The number of doors cannot be lowered from 3 to 2 -and the number of goats therefore from 2 to 1 -because it then becomes impossible to always open a door revealing a goat. By using Equation (4), it can be determined that the chances of getting the car by switching are as follows.

$$
\frac{c(d-1)}{d(d-1-o)}=\frac{1(3-1)}{3(3-1-1)}=\frac{2}{3}
$$

Moreover, the probability of getting the car is increased through switching by a factor of $\frac{d-1}{d-1-o}$, in this case of $\frac{1(3-1)}{3(3-1-1)}=2$.

Because there is only 1 car, it is possible to use Equation (5) in §4.1. The probability of getting the car by switching doors is then determined as follows.

$$
\frac{g}{d(g-o)}=\frac{2}{3(2-1)}=\frac{2}{3}
$$


In any variations on the Monty Hall problem, the numbers of doors hiding cars and goats and opened doors can only be increased. Increasing the doors hiding cars increases the probability of initially picking a car and therefore also of getting a car by not switching. Increasing doors hiding goats decreases that same probability. But neither will increase the probability of getting a car by switching as opposed to not switching. That probability is increased only by increasing the number of opened doors.

\subsubsection{Changing the Number of Doors $(d)$ and Doors Opened to Reveal Goats (o)}

The most characteristic effect of the Monty Hall problem and its variations is the way in which opening doors to reveal goats increases the probability of getting a car by switching doors. What is more, the increase of the probability of getting a car by switching doors grows as ever more and more doors are opened to reveal goats. But this increase can only grow on condition that there are more doors hiding goats in the first place.

For example, the number of doors hiding goats could be increased to 9 and the total number of doors therefore to 10 . If only 1 door is opened to reveal a goat, the probability of getting the car by switching doors $\left(C_{s}\right)$ is, in light of Equation (4) in $\S 4.1$, as follows, given 1 car ( $c$ ), 10 doors $(d)$, and 1 opened door $(o)$.

$$
\frac{c(d-1)}{d(d-1-o)}=\frac{1(10-1)}{10(10-1-1)}=\frac{9}{80} \text { or } 11.25 \%
$$

Because there is only 1 car, it is possible to use Equation (5) in $\S 4.1$. The probability of getting the car by switching doors is then determined as follows.

$$
\frac{g}{d(g-o)}=\frac{9}{10(9-1)}=\frac{9}{80}
$$

The probability of getting the car by not switching is $1 / 10$ or $10 \%$. The increase in the probability of getting the car by switching is only $1.25 \%$. The difference between switching and not switching would only become noticeable as the process of picking a door by switching doors is repeated over and over again. But as the opened doors $(o)$ increase in number, so does the probability of getting the car by switching doors. If there are 2 doors, the probability is as follows.

$$
\frac{g}{d(g-o)}=\frac{9}{10(9-2)}=\frac{9}{70} \text { or about } 12.6 \%
$$

As 6 more doors hiding goats are opened one by one until only 1 unopened door hiding either a car or a goat remains, the probability of getting the car by switching doors gradually increases as follows: from $9 / 60$ (15\%) to $9 / 50(18 \%)$ to $9 / 40(22.5 \%)$ to $9 / 30$ (30\%) to $9 / 20$ (45\%) and finally to $9 / 10$ (90\%). When only 1 door is left unopened besides the initially picked door, the chances of getting the car are 9 times greater when switching doors than when not switching doors and one has a $90 \%$ chance if one switches. What is more, the probability of getting the car by switching doors no less than doubles when, of 2 doors remaining unopened besides the picked door, 1 is opened and only 1 door is left to which one can switch.

If there are 1000 doors of which 1 hides a car and 999 hide a goat and 998 doors are opened to reveal goats, the chance of getting the car by switching to the 1 door remaining unopened is as follows.

$$
\frac{g}{d(g-o)}=\frac{999}{1000(999-998)}=\frac{999}{1000}
$$

One has a $99.9 \%$ chance of getting the car by switching doors.

\subsubsection{Changing the Number of Cars (c)}

The examples provided have so far illustrated changing the number of doors hiding goats $(g)$ and changing the number of opened doors $(o)$. The main aim of the examples was to illustrate the effect of opening doors in terms of probability. Towards that end, the number of doors being opened to reveal goats had to be increased. But this number cannot be increased without increasing the number of doors hiding goats. That still leaves changing the number of cars to be illustrated.

As was noted above ( $\$ 4.1$ end), when there is $1 \mathrm{car}$, there is one scenario, and when there is more than 1 car, there are two scenarios. As will be seen below, when there are two scenarios, the probability of getting a car by switching doors consists of a sum of two terms. When there is only one scenario, there is only one term.

Let us assume that $c=6, g=4, d=10$. The probability of initially picking a door hiding car and therefore also of getting a car by not switching doors is $6 / 10$ or $60 \%$. The probability of getting a car by switching doors increases as follows when first 1 , then 2 , and finally 3 doors are opened to reveal a goat.

$$
\begin{aligned}
& \text { opening } 1 \text { door: } \frac{6(10-1)}{10(10-1-1)}=\frac{54}{80} \text { or } 67.5 \% \\
& \text { opening } 2 \text { doors: } \frac{6(10-1)}{10(10-1-2)}=\frac{54}{70} \text { or about } 71.1 \% \\
& \text { opening } 3 \text { doors: } \frac{6(10-1)}{10(10-1-3)}=\frac{54}{60} \text { or } 90 \%
\end{aligned}
$$




\subsection{Probability Theory as a Mathematical Discipline That Combines the Digital or Non-quantitative with the Quantitative}

The design of $\S \S 4.4-7$ is to clarify how Equation (4) (§4.1) has been obtained. This Equation makes it possible not only to determine the probability of getting a car by switching doors under the precise conditions stipulated in the Monty Hall problem (see above) but also to extend and generalize the Monty Hall problem to any number of doors, cars, and doors that are opened to reveal a goat. However, getting a car is only one possible outcome. The other outcome is not getting the car. Equation (4) is therefore only one component of a larger mathematical problem. To ensure an adequate analysis and a true appreciation of the Monty Hall problem itself, it will be necessary to map the mathematical structure of the larger problem. The larger problem concerns all possible outcomes. The total probability of all outcomes is 1 or $100 \%$. One or the other outcome of all possible outcomes must always be the case.

But first, to ensure an even deeper appreciation of the Monty Hall problem on an even more general level, it will be useful to highlight a most peculiar general property of the type of mathematics needed to analyze the problem and its extensions, namely probability theory. In that regard, no one more than Boole has taken pains to emphasize - and in fact hardly anyone has in addition to him-that probability theory is a mathematical discipline straddling the digital or non-quantitative and the quantitative.

The mathematical anatomy of the larger problem of which the Monty Hall is a specific case hence has two components, a digital or non-quantitative component and a quantitative component. The relation between the digital component and the quantitative component is such that the digital component provides a skeleton that is fleshed out by the quantitative component.

\subsection{The Digital or Non-quantitative Component in the Mathematical Mapping of the Monty Hall Problem and Its Extensions}

The larger problem of which the Monty Hall problem presents just one specific case revolves entirely around the four possible combination events in which either a first event or its supplement or negation can be followed by either a second event or its supplement or negation. In the specific case of the Monty Hall problem, the probability of two of the four possible combination events is zero.
Two classes, whether of things or of events, and their supplements produce exactly four combination classes (§2.6). Three events and their supplements would produce exactly eight combination classes, and so on. This structure is digital or non-quantitative. One might rightly object that at least the number of the combination classes is quantitative. Strictly speaking, therefore, it is only the exploitation of the contrast between a class and its supplement and the combination of classes and their supplements into combination classes that is digital.

Certain combinations of two classes and their supplements are invalid. Combining a class with itself produces just that class (§2.1) and is therefore not a valid combination. Combining a class with its supplement is not possible because, according to the fundamental axiom of thought, something cannot at the same time be and not be something (§2.8).

In the case of the Monty Hall problem and any extensions thereof, the first event is initially $(i)$ picking a door hiding a car $(C)$, denoted here by $C_{i}$. The first event can also be defined as failing to pick a door hiding a goat or a non-car. The supplement or negation of the first event is initially ( $i$ ) failing to pick a door hiding a car $(\bar{C})$, denoted here by $\bar{C}_{i}$. The supplement may also be described as picking a door hiding a non-car. The second event is picking a door hiding a car $(C)$ by switching $(s)$ doors and is denoted here by $C_{s}$. The supplement or negation of the second event is failing to pick a door hiding a car $(\bar{C})$ by switching (s) doors and is denoted here by $\bar{C}_{s}$. In sum, there are exactly four digital combination classes (of events), the following.

(1) $C_{i} \times C_{s}$

(2) $C_{i} \times \bar{C}_{s}$

(3) $\bar{C}_{i} \times C_{s}$

(4) $\bar{C}_{i} \times \bar{C}_{s}$

The total probability of the occurrence of these four digital combination events is 1 or $100 \%$. That means that one of these four combination events must always be the case. The four digital combination classes are also mutually exclusive. Combining them would result in combinations of a class and its supplement. As has already been noted, according to the fundamental axiom of thought, such combinations are impossible. Something cannot at the same time be and not be something.

Of the four digital combination classes, two have the desired outcome of picking a car by switching doors $\left(C_{S}\right)$, namely (1) and (3). It is on these two combination classes that the study of the Monty Hall problem and any extensions thereof focuses (see $\S 4.7$ below). But the problem can only be mathematically appreciated to its full extent by considering all possible outcomes, whose combined probability is 1 or $100 \%$. 


\subsection{The Quantitative Component in the Mathematical Mapping of the Monty Hall Problem and Its Extensions}

\subsubsection{Preamble: Boole's “ $\times$ ” as an Expression of How the Digital and the Quantitative Complement One Another as Two Facets of a Single Phenomenon}

At the center of the mathematical structure of the larger problem of which the Monty Hall problem presents just one specific case stand four mutually exclusive digital combination classes of events. In Boole's algebra, they are denoted by

$$
C_{i} \times C_{s}, C_{i} \times \bar{C}_{s}, \bar{C}_{i} \times C_{s} \text {, and } \bar{C}_{i} \times \bar{C}_{s} .
$$

As a notation of digital combination classes, the symbol " $x$ " represents the Boolean AND-operator, which is used for example when one searches for all that is both Paris and hotels on the Internet.

However, in Boole's algebra, the notations $C_{i} \times C_{s}$, $C_{i} \times \bar{C}_{s}, \bar{C}_{i} \times C_{s}$, and $\bar{C}_{i} \times \bar{C}_{s}$ can also stand for the numerical probabilities of the occurrence of the four combination classes. The symbol " $\times$ " then functions as the multiplication sign of quantitative mathematics. Boole's notation system therefore most felicitously represents probability as a single phenomenon that exhibits digital or non-quantitative properties and quantitative properties inextricably linked to one another.

The quantitative probability of a combination class of events involving two events is obtained by first calculating the probability of the first event, then calculating the probability of the second event, and finally multiplying the two probabilities with one another.

In calculating the probability of the second event, it needs to be taken in account that the second event may be dependent on the first event according to the definition of dependence in classical probability theory. The dependence is such that the occurrence of the first event creates a new situation that influences the calculation of the probability of the second event. In other words, the second event takes place in a environment different from the one in which the first event takes place. And it is the first event that changed the environment.

\subsubsection{The Probability of Digital Combination Event}

$$
C_{i} \times C_{s}: \frac{c}{d} \times \frac{c-1}{d-1-o}
$$

To establish the probability of $C_{i} \times C_{s}$, the probability of the first event $C_{i}$ initially (i) picking a door hiding a car (C), needs to be determined first. This probability is the ratio of all the cars to all the doors, which can be denoted by $c / d$. If there is 1 car and there are 3 doors, the chance of picking the door hiding the car is 1 in 3 .
Next is establishing the probability of the second event $C_{s}$, picking a door hiding a car $(C)$ by switching doors $(s)$. This probability is also a ratio of cars to doors. Since a car has already been picked in the first event, the number of cars needs to be reduced by 1 to $c-1$. And since one cannot pick again the door picked in the first event because one needs to switch, the number of doors needs to be reduced by 1 to $d-1$. As was already noted above, the number of picked doors, 1 , is not denoted algebraically by, say, $p$ because the formula being developed here is only valid when 1 door is picked, that is, when $p=1$. Furthermore, since one cannot pick any doors being opened to reveal a goat, $d-1$ needs to be reduced further by the number of opened doors (o) to $d-1-o$. The probability of $C_{s}$ will therefore be $\frac{c-1}{d-1-o}$ as a ratio of cars to doors.

The combined probability of $C_{i} \times C_{s}$ will be $\frac{c}{d} \times \frac{c-1}{d-1-o}$. In the case of the Monty Hall problem, the probability in question is as follows.

$$
\frac{1}{3} \times \frac{1-1}{3-1-1}=\frac{1}{3} \times \frac{0}{1}=\frac{1}{3} \times 0=0
$$

This probability is zero. It is not possible to pick the door hiding the car by switching doors if one has already initially picked the car because there is only 1 car.

\subsubsection{The Probability of Digital Combination Event}

$$
C_{i} \times \bar{C}_{s}: \frac{c}{d} \times \frac{g-o}{d-1-o}
$$

As regards the probability of $C_{i} \times \bar{C}_{s}$, the probability of the first event $C_{i}$ has already been determined to be $c / d(\S 4.6 .2)$.

Next is establishing the probability of the second event $\bar{C}_{s}$, picking a door hiding a goat or non-car $(\bar{C})$ by switching doors (s). This probability is a ratio of goats to doors. Since no goat has been picked in the first event, the number of goats does not need to be reduced in this respect. However, since one cannot pick any doors being opened to reveal a goat, the goats in those doors cannot be picked either and the number of the goats $(g)$ needs to be reduced by the number of opened doors (o) to $g-o$. Furthermore, again because one cannot pick any doors being opened to reveal a goat, the number of doors available for being picked $(d-1)$ needs to be reduced as well by the number of opened doors $(o)$, namely to $d-1-o$. The probability of $C_{\mathrm{s}}$ will therefore be

$\frac{g-o}{d-1-o}$ as a ratio of goats to doors.

The combined probability of $C_{i} \times \bar{C}_{s}$ will be 
$\frac{c}{d} \times \frac{g-o}{d-1-o}$. In the case of the Monty Hall problem, the probability in question is as follows.

$$
\frac{1}{3} \times \frac{2-1}{3-1-1}=\frac{1}{3} \times \frac{1}{1}=\frac{1}{3} \times 1=\frac{1}{3}
$$

In one third of all possible cases, one will initially pick a door hiding a car and then pick a door hiding a goat by switching doors.

\subsubsection{The Probability of Digital Combination Event}

$$
\bar{C}_{i} \times C_{s}: \frac{g}{d} \times \frac{c}{d-1-o}
$$

To establish the probability of $\bar{C}_{i} \times C_{s}$, the probability of the first event $\bar{C}_{i}$, initially (i) picking a door hiding a goat or non-car $(\bar{C})$, needs to be determined first. This probability is a ratio of all the goats to all the doors, which can be denoted by $g / d$. If there are 2 goats and there are 3 doors, the chance of picking a door hiding a goat is 2 in 3.

Next is establishing the probability of the second event $C_{s}$, picking a door hiding a car $(c)$ by switching doors (s). This probability is a ratio of cars to doors. Since no car has been picked in the first event, the number of cars (c) does not need to be reduced. But since one cannot pick again the door picked in the first event because one needs to switch, the number of doors needs to be reduced by 1 to $d-1$. Furthermore, since one cannot pick any doors being opened to reveal a goat, $d-1$ needs to be reduced further by the number of opened doors $(o)$ to $d-1-o$. The probability of $C_{s}$ will therefore be

$\frac{c}{d-1-o}$ as a ratio of cars to doors.

The combined probability of $\bar{C}_{i} \times C_{s}$ will be $\frac{g}{d} \times \frac{c}{d-1-o}$. In the case of the Monty Hall problem, the probability in question is as follows.

$$
\frac{2}{3} \times \frac{1}{3-1-1}=\frac{2}{3} \times \frac{1}{1}=\frac{2}{3} \times 1=\frac{2}{3}
$$

In two thirds of all possible cases, one will initially pick a door hiding a goat and then pick a door hiding the car by switching doors.

\subsubsection{The Probability of Digital Combination Event}

$$
\bar{C}_{i} \times \bar{C}_{s}: \frac{g}{d} \times \frac{g-1-o}{d-1-o}
$$

As regards the probability of $\bar{C}_{i} \times \bar{C}_{s}$, the probability of the first event $\bar{C}_{i}$ has already been determined to be $g / d(\S 4.6 .4)$.
Next is establishing the probability of the second event $\bar{C}_{s}$, picking a door hiding a goat or non-car $(\bar{C})$ by switching doors $(s)$. This probability is a ratio of goats to doors. Since a goat has been picked in the first event, the number of goats needs to be reduced by 1 to $g-1$. Furthermore, since one cannot pick any doors opened to reveal a goat, the goats in those doors cannot be picked either and the number of the goats needs to be reduced further by the number of opened doors (o) to $g-1-o$. Furthermore, also because one cannot pick any doors being opened to reveal a goat, the number of doors available for being picked ( $d-1)$ needs to be reduced as well by the number of opened doors $(o)$, namely to

$d-1-o$. The probability of $\bar{C}_{s}$ will be $\frac{g-1-o}{d-1-o}$ as a ratio of goats to doors.

The combined probability of $\bar{C}_{i} \times \bar{C}_{s}$ will be $\frac{g}{d} \times \frac{g-1-o}{d-1-o}$. In the case of the Monty Hall problem, the probability in question is as follows.

$$
\frac{2}{3} \times \frac{2-1-1}{3-1-1}=\frac{2}{3} \times \frac{0}{1}=\frac{2}{3} \times 0=0
$$

This probability is zero. There are 2 goats. It is not possible to pick a door hiding a goat by switching doors if one has already initially picked a goat because the other door hiding a goat is opened and the second goat is no longer available for picking.

\subsubsection{The Combined Probability of All Digital Combination Events: 1 or $\mathbf{1 0 0 \%}$}

In a digital perspective, two classes and their supplements partition the universe or all that one could possibly think about into four combination classes (§2.6). The universe that is being considered in any partition often exhibits unspoken or explicit limits and is therefore not quite all that one could think about (§2.7). In a quantitative perspective, the numerical probabilities of all four combination events must add up to 1 or $100 \%$. One or the other of the four combination classes must be the case and the four combination classes may take place with different degrees of probability. Accordingly, the following Equation applies. The notation is Boole's.

$$
\begin{aligned}
& \frac{c}{d} \times \frac{c-1}{d-1-o}+\frac{c}{d} \times \frac{g-o}{d-1-o}+\frac{g}{d} \times \frac{c}{d-1-o} \\
& +\frac{g}{d} \times \frac{g-1-o}{d-1-o}=1
\end{aligned}
$$

Because probability exhibits simultaneously a digital or non-quantitative facet and a quantitative facet, this Equation can be read in two different ways (§4.6.1). If “ $x$ " is interpreted as the Boolean AND-operator, the reading is digital and the four terms are combination 
classes of events, each characterized by the joint occurrence $(x)$ of two events. If “ $x$ " is interpreted as the multiplication sign of quantitative mathematics, the four products $(\times)$ represent the numerical probabilities of the occurrence of each of the combination events.

\subsection{The Probability of the Two Digital Combination Events Producing the Desired Outcome, Getting the Car, by Switching Doors, namely $C_{i} \times C_{s}$ and $\bar{C}_{i} \times C_{s}$}

The Monty Hall problem and any extensions thereof revolve around a challenge with a desired outcome, namely getting a car. The question is whether the desired outcome can be achieved with greater or lesser probability by switching doors. The focus is therefore on determining the probability of all the cases in which one gets the car by switching doors. It appears that only two of the four possible combination events discussed above (§4.6) have getting a car by switching doors as the outcome, namely $C_{i} \times C_{s}$ and $\bar{C}_{i} \times C_{s} \quad(\S \S 4.6 .2$ and 4.6.4). The combined probability of these two combination events is as follows ( $\S 4.6 .2$ and 4.6.4).

$$
\frac{c}{d} \times \frac{c-1}{d-1-o}+\frac{g}{d} \times \frac{c}{d-1-o}
$$

If there is only 1 car, then $c-1=0$ and therefore $\frac{c-1}{d-1-o}=0$ and $\frac{c}{d} \times \frac{c-1}{d-1-o}=0$. The probability of getting a car by switching is then as follows.

$$
\frac{g}{d} \times \frac{c}{d-1-o}
$$

The two products making up expression (14) have the same denominator. Expression (14) can therefore be rewritten as follows.

$$
\frac{c(c-1)+g c}{d(d-1-o)}
$$

And, in light of the common factor $c$, also as follows.

$$
\frac{c(c-1+g)}{d(d-1-o)}
$$

And further as follows.

$$
\frac{c(c+g-1)}{d(d-1-o)}
$$

And since $c+g=d$, the following expression is also equivalent.

$$
\frac{c(d-1)}{d(d-1-o)}
$$

This is the probability that switching doors will pay off by getting a car, as stated in Equation (4) in §4.1.

\section{Extension and Generalization of the Monty Hall Problem to Any Number of Picked Doors $(p)$}

\section{1. "Doubling” the Monty Hall Problem}

Very many are the ways in which the Monty Hall problem can be extended. One might for example consider cases in which one can pick three or more types of things hiding behind doors, cars and goats and other types of things. Exploring more of these extensions remains desirable. But what can be done within the confines of the present paper is limited. Moreover, a principal aim of the present paper is highlighting the digital component of the analysis of the problem and its extensions in its relation to digitality as a fundamental component of human cognition. A discussion of countless extensions of the Monty Hall problem would probably not shed much additional light on the fundamental assumption made here as a working hypothesis, namely that rational thought and language is profoundly digital. In what follows, the analysis of extensions will be limited to cases in which more than one door can be picked.

One specific case in which more than one door is picked involves "doubling” the Monty Hall problem in all its characteristics. Accordingly, there would be 2 cars instead of 1, 4 goats instead of 2, 6 doors instead of 3, and 2 doors would be opened to reveal goats instead of 1 . Furthermore, the question would now be: If one picks 2 doors that remained closed and 2 doors are opened to reveal goats, should one switch to the 2 remaining unopened doors to improve one's chances of getting 2 cars and, if so, by how much would one improve one's chances?

\subsection{The Increase in Digital Complexity}

Because probability is a phenomenon exhibiting both digital or non-quantitative and quantitative properties (§§3.1 and 4.6.1), making the number of picked doors into a variable will add complexity to the analysis of the extended Monty Hall problem. As regards the digital complexity, the original Monty Hall problem and the extensions to any number of doors, cars, and opened doors discussed above involve only digital combination events. The additional extension to any number of picked doors involves digital combinations of digital combination events.

Consider the case in which 2 doors are picked initially, as in the "doubling” of the Monty Hall problem (§5.1). In the original Monty Hall problem and its extension to 
any number of doors, cars, and opened doors discussed above, the initial pick of a door and the pick of a door by switching doors are both single events.

By contrast, when 2 doors are initially picked and 2 doors are picked after switching, both the initial pick and the pick by switching are composite. Both consist themselves of combination events. There are 2 initial picks that follow one another in sequence and 2 picks by switching that also follow one another in sequence.

In both the 2 initial picks and the 2 picks by switching, two classes of events and their supplements generate four digital combination classes (§2.6). As regards the 2 initial picks, the two classes of events are picking a car $(C)$ in the first $(f)$ initial $(i)$ pick $\left(C_{f i}\right)$ and picking a car $(C)$ in the second $(s)$ initial $(i)$ pick $\left(C_{s i}\right)$. The two supplement classes are failing to pick a $\operatorname{car}(\bar{C})$ in the first $(f)$ initial $(i)$ pick $\left(\bar{C}_{f i}\right)$, or picking a goat, and failing to pick a car $(\bar{C})$ in the second $(s)$ initial $(i)$ pick $\left(\bar{C}_{s i}\right)$, or picking a goat. Accordingly, the four digital combination classes characterizing just the 2 initial picks are as follows: $C_{f i} \times C_{s i}, C_{f i} \times \bar{C}_{s i}, \bar{C}_{f i} \times C_{s i}$, and $\bar{C}_{f i} \times \bar{C}_{s i}$.

As regards the 2 picks by switching doors after doors have been opened to reveal goats, the two classes of events are picking a car $(C)$ in the first $(f)$ pick by switching $(s)\left(C_{f s}\right)$ and picking a car $(C)$ in the second $(s)$ pick by switching $(s)\left(C_{s s}\right)$. The supplement of the first event is failing to pick a car $(\bar{C})$ in the first $(f)$ pick by switching $(s)\left(\bar{C}_{f s}\right)$, or picking a goat. The supplement of the second event is failing to pick a car $(\bar{C})$ in the second ( $s$ ) pick by switching $(s)\left(\bar{C}_{s s}\right)$, or picking a goat. Accordingly, the four digital combination classes characterizing the 2 picks by switching doors are as follows: $C_{f s} \times C_{s s}, C_{f s} \times \bar{C}_{s s}, \bar{C}_{f s} \times C_{s s}$, and $\bar{C}_{f s} \times \bar{C}_{s s}$.

\subsection{The 16 Digital Combinations of the "Doubled" Monty Hall Problem}

Each of the four combination events of the initial picks is combined with each of the four combination classes of the picks by switching. The result is 16 combinations of combination events. The 16 combinations are listed below along with the numerical probability of their occurrence.

$$
\begin{aligned}
& c=\text { cars } p=\text { doors picked in the initial picks } \\
& g=\text { goats } \begin{array}{r}
o \\
=
\end{array} \\
& \text { doors opened to reveal a goat after the } \\
& \text { intial picks }
\end{aligned}
$$$$
\text { (1) }
$$

$$
\begin{aligned}
& \left(C_{f i} \times C_{s i}\right) \times\left(C_{f s} \times C_{s s}\right): \\
& \left(\frac{c}{d} \times \frac{c-1}{d-1}\right) \times\left(\frac{c-2}{d-p-o} \times \frac{c-3}{d-p-o-1}\right)=0
\end{aligned}
$$

(2)

$$
\begin{aligned}
& \left(C_{f i} \times \bar{C}_{s i}\right) \times\left(C_{f s} \times C_{s s}\right): \\
& \left(\frac{c}{d} \times \frac{g}{d-1}\right) \times\left(\frac{c-1}{d-p-o} \times \frac{c-2}{d-p-o-1}\right)=0
\end{aligned}
$$

(3)

$$
\begin{aligned}
& \left(\bar{C}_{f i} \times C_{s i}\right) \times\left(C_{f s} \times C_{s s}\right): \\
& \left(\frac{g}{d} \times \frac{c}{d-1}\right) \times\left(\frac{c-1}{d-p-o} \times \frac{c-2}{d-p-o-1}\right)=0
\end{aligned}
$$

$$
\begin{aligned}
& \left(\bar{C}_{f i} \times \bar{C}_{s i}\right) \times\left(C_{f s} \times C_{s s}\right): \\
& \left(\frac{g}{d} \times \frac{g-1}{d-1}\right) \times\left(\frac{c}{d-p-o} \times \frac{c-1}{d-p-o-1}\right) \\
& =\left(\frac{4}{6} \times \frac{3}{5}\right) \times\left(\frac{2}{2} \times \frac{1}{1}\right)=\frac{6}{15}
\end{aligned}
$$

(5)

$$
\begin{aligned}
& \left(C_{f i} \times C_{s i}\right) \times\left(C_{f s} \times \bar{C}_{s s}\right): \\
& \left(\frac{c}{d} \times \frac{c-1}{d-1}\right) \times\left(\frac{c-2}{d-p-o} \times \frac{g-o}{d-p-o-1}\right)=0
\end{aligned}
$$

(6)

$$
\begin{aligned}
& \left(C_{f i} \times \bar{C}_{s i}\right) \times\left(C_{f s} \times \bar{C}_{s s}\right): \\
& \left(\frac{c}{d} \times \frac{g}{d-1}\right) \times\left(\frac{c-1}{d-p-o} \times \frac{g-o-1}{d-p-o-1}\right) \\
& =\left(\frac{2}{6} \times \frac{4}{5}\right) \times\left(\frac{1}{2} \times \frac{1}{1}\right)=\frac{2}{15}
\end{aligned}
$$

$$
\begin{aligned}
& \left(\bar{C}_{f i} \times C_{s i}\right) \times\left(C_{f s} \times \bar{C}_{s s}\right): \\
& \left(\frac{g}{d} \times \frac{c}{d-1}\right) \times\left(\frac{c-1}{d-p-o} \times \frac{g-o-1}{d-p-o-1}\right) \\
& =\left(\frac{4}{6} \times \frac{2}{5}\right) \times\left(\frac{1}{2} \times \frac{1}{1}\right)=\frac{2}{15}
\end{aligned}
$$

$$
\begin{aligned}
& \left(\bar{C}_{f i} \times \bar{C}_{s i}\right) \times\left(C_{f s} \times \bar{C}_{s s}\right): \\
& \left(\frac{g}{d} \times \frac{g-1}{d-1}\right) \times\left(\frac{c}{d-p-o} \times \frac{g-o-2}{d-p-o-1}\right)=0
\end{aligned}
$$

$$
\begin{aligned}
& \left(C_{f i} \times C_{s i}\right) \times\left(\bar{C}_{f s} \times C_{s s}\right): \\
& \left(\frac{c}{d} \times \frac{c-1}{d-1}\right) \times\left(\frac{g-o}{d-p-o} \times \frac{c-2}{d-p-o-1}\right)=0
\end{aligned}
$$


(10)

$$
\begin{aligned}
& \left(C_{f i} \times \bar{C}_{s i}\right) \times\left(\bar{C}_{f s} \times C_{s s}\right): \\
& \left(\frac{c}{d} \times \frac{g}{d-1}\right) \times\left(\frac{g-o-1}{d-p-o} \times \frac{c-1}{d-p-o-1}\right) \\
& =\left(\frac{2}{6} \times \frac{4}{5}\right) \times\left(\frac{1}{2} \times \frac{1}{1}\right)=\frac{2}{15}
\end{aligned}
$$

(11)

$$
\begin{aligned}
& \left(\bar{C}_{f i} \times C_{s i}\right) \times\left(\bar{C}_{f s} \times C_{s s}\right): \\
& \left(\frac{g}{d} \times \frac{c}{d-1}\right) \times\left(\frac{g-o-1}{d-p-o} \times \frac{c-1}{d-p-o-1}\right) \\
& =\left(\frac{4}{6} \times \frac{2}{5}\right) \times\left(\frac{1}{2} \times \frac{1}{1}\right)=\frac{2}{15}
\end{aligned}
$$

$$
\begin{aligned}
& \left(\bar{C}_{f i} \times \bar{C}_{s i}\right) \times\left(\bar{C}_{f s} \times C_{s s}\right): \\
& \left(\frac{g}{d} \times \frac{g-1}{d-1}\right) \times\left(\frac{g-o-2}{d-p-o} \times \frac{c}{d-p-o-1}\right)=0
\end{aligned}
$$

(13)

$$
\begin{aligned}
& \left(C_{f i} \times C_{s i}\right) \times\left(\bar{C}_{f s} \times \bar{C}_{s s}\right): \\
& \left(\frac{c}{d} \times \frac{c-1}{d-1}\right) \times\left(\frac{g-o}{d-p-o} \times \frac{g-o-1}{d-p-o-1}\right) \\
& =\left(\frac{2}{6} \times \frac{1}{1}\right) \times\left(\frac{2}{2} \times \frac{1}{1}\right)=\frac{1}{15}
\end{aligned}
$$

(14)

$$
\begin{aligned}
& \left(C_{f i} \times \bar{C}_{s i}\right) \times\left(\bar{C}_{f s} \times \bar{C}_{s s}\right): \\
& \left(\frac{c}{d} \times \frac{g}{d-1}\right) \times\left(\frac{g-o-1}{d-p-o} \times \frac{g-o-2}{d-p-o-1}\right)=0
\end{aligned}
$$

$$
\begin{aligned}
& \left(\bar{C}_{f i} \times C_{s i}\right) \times\left(\bar{C}_{f s} \times \bar{C}_{s s}\right): \\
& \left(\frac{g}{d} \times \frac{c}{d-1}\right) \times\left(\frac{g-o-1}{d-p-o} \times \frac{g-o-2}{d-p-o-1}\right)=0
\end{aligned}
$$

$$
\begin{aligned}
& \left(\bar{C}_{f i} \times \bar{C}_{s i}\right) \times\left(\bar{C}_{f s} \times \bar{C}_{s s}\right): \\
& \left(\frac{g}{d} \times \frac{g-1}{d-1}\right) \times\left(\frac{g-o-2}{d-p-o} \times \frac{g-o-3}{d-p-o-1}\right)=0
\end{aligned}
$$

The probability of 10 combinations of combination events is zero. In (1), (2), (3), (5), and (9), cars are picked in 3 or 4 of the 4 picks, but there are only 2 cars. In (8), (12), (14), (15), and (16), goats are picked in 3 or 4 of the 4 picks, but it is not possible to pick more than 2 in total. There are two scenarios. First, in (8), (12), and (16), 2 goats are initially picked. When 2 doors are then opened to reveal goats, there are no goats left to pick. Yet, 1 goat is picked according to (8), (12), and (16).
This is not possible. Second, in (14) and (15), 1 goat is initially picked. When 2 doors are then opened to reveal goats, only 1 goat is available for picking. Yet, 2 goats are picked according to (14) and (15). This is also not possible.

\subsection{Dependence}

Each combination in $\S 5.3$ consists of a sequence of four events, with the second event being dependent on the first, the third on the first two, and the fourth on the first three. For example, in (4), a door hiding a goat is initially picked in the first event. The chance of picking a goat is $c / d$. In the second event, a door hiding a goat is again picked. But the number of the goats and the doors has been reduced by 1 in the first event. The chance of again picking a goat in the second event is therefore $(c-1) /(d-1)$. In the third event, a car is picked. But the number of doors has been reduced by the 2 doors that are picked in the first and second events $(p)$, though not the number of cars as no car has been picked yet. In addition, the number of doors is reduced by 2 as 2 doors are opened to reveal a goat $(o)$. The chance of picking a car is therefore $(c-2) /(d-p-o)$. Finally, in the fourth event, a car is again picked. But, the number of cars has been reduced by 1 . So has again the number of doors. The chance of picking a car is therefore

$(c-3) /(d-p-o-1)$. When goats are picked by switching doors, that is, in the third and fourth component events, the number of goats can be reduced not only by goats that are picked but also by goats revealed by opening doors $(o)$.

\subsection{The Probability of a Successful Outcome by Switching Doors in the "Doubled" Monty Hall Problem}

Is one more likely or less likely of getting 2 cars by switching doors than by not switching doors after 2 doors have been opened to reveal goats if there are 2 cars, 4 goats, and 6 doors? To answer this question, the probabilities of getting the 2 cars by not switching and by switching need to be compared.

The probability of getting the 2 cars by not switching is the same as the probability of initially getting 2 cars by picking 2 doors. The probability of picking 1 of the cars at first pick is $c / d$ or $2 / 6$ or $1 / 3$. If one was successful in picking a car at first pick, then not only the number of the doors but also the number of the cars is reduced by 1 . The probability of picking a car again at second pick is therefore $(c-1) /(d-1)$ or $(2-1) /(6-1)$ or $1 / 5$. The probability of picking both cars in the first 2 picks, then, is therefore $1 / 3 \times 1 / 5$ or $1 / 15$ or about $6.6 \%$.

As for the probability of getting the 2 cars by switching 
from the 2 doors initially picked to the 2 only doors that remain closed after 2 doors have been opened to reveal 2 goats, 2 cars are picked in only 1 of the 16 digital combinations listed in $\$ 5.3$, namely (4). The probability of the occurrence of this combination is $6 / 15$ or $2 / 5$ or $40 \%$. Remarkably, one can only get the 2 cars by switching doors if one had originally picked 2 doors hiding goats or no cars at all.

\subsection{Comparison of the Original and the "Doubled" Monty Hall Problems}

In the original Monty Hall problem, one is more likely to get the car than not getting it after switching doors. The probability of getting the car is $2 / 3$. By contrast, in "doubling" the problem, one is less likely to get the 2 cars than not getting them after switching doors. The probability of getting them is 6/15. On the other hand, in the original Monty Hall problem, one doubles one's chances to get the car from $1 / 3$ to $2 / 3$ by switching doors. By contrast, in “doubling” the problem, one's chances of getting both cars increase sixfold from 1/15 to $6 / 15$.

\subsection{Compressing Probability as an Effect of Opening Doors to Reveal Goats}

As always, opening doors to reveal goats has the effect of compressing or condensing a greater probability to fewer doors (§3.3). There is a probability of only 1/15 of getting both cars by initially picking 2 doors (§5.5). However, there is a probability of $6 / 15$ or $40 \%$ that the 2 cars are both hiding behind 2 of the other 4 doors that one has not picked. This number is obtained as follows.

There are 15 different ways in which the 2 cars can be hiding behind the 6 doors: behind doors 1 and 2, doors 1 and 3, doors 1 and 4, doors 1 and 5, doors 1 and 6, doors 2 and 6, doors 2 and 3, doors 2 and 4, doors 2 and 5, doors 2 and 6, doors 3 and 4, doors 3 and 5, doors 3 and 6 , doors 4 and 5 , doors 4 and 6 , and doors 5 and 6 . Let us assume that the doors picked initially are 1 and 2. In 6 out of a total of 15 ways, or $40 \%$, the 2 cars are hiding behind the 4 other doors, namely doors 3 to 6 . They are the last 6 locations in the list just provided. In 9 of 15 ways, at least 1 car is hiding behind either door 1 or door 2 or both.

\subsection{Denominator and Numerator of the Probabilities Involved in the "Doubled" Monty Hall Problem}

\subsubsection{Denominator}

All 16 digital combination events listed in §5.3 exhibit the same denominator. Furthermore, as the number of picks increases, the denominator will morph into

$$
\begin{aligned}
& d(d-1)(d-2)(d-3) \cdots \times(d-p-o) \times(d-p-o-1) \\
& \times(d-p-o-2) \times(d-p-o-3) \times \cdots
\end{aligned}
$$

This progression is obviously factorial, as expressed by “!”. For any number of doors $(d)$, opened doors $(o)$, and picked doors $(p)$, the numerator will therefore be as follows.

$$
\frac{d !}{(d-p) !} \times \frac{(d-p-o) !}{[(d-p-o)-p] !}
$$

The details exceed the scope of the present paper. Suffice it to note that 0 !, which is obtained when $(d-p-o)-p=0$, is not the same as zero or nothing but rather signifies the absence of any additional factor.

\subsubsection{Numerator}

As regards the numerator of the 16 combination classes listed in §5.3, an evaluation is necessary in terms of what is sought. The question was asked before how much one improves one's chances of getting both doors by switching doors. But what if one took satisfaction with getting just 1 car? How does one improve one's chances in that case by switching both doors? One's initial chances of getting at least 1 car by picking 2 doors is the combined probability of the three combined events of picking first a car and then again a car, of picking first a car and then a goat, and of picking first a goat and then a car, or

$\frac{c}{d} \times \frac{c-1}{d-1}+\frac{c}{d} \times \frac{g}{d-1}+\frac{g}{d} \times \frac{c}{d-1}$, in this case $\frac{2}{6} \times \frac{1}{5}+\frac{2}{6} \times \frac{4}{5}+\frac{4}{6} \times \frac{2}{5}=\frac{9}{15}$. As regards switching, the outcome of five of the 16 combination classes listed above includes getting at least 1 car. They are combination classes (4), (6), (7), (10), and (11). Their combined probability is $14 / 15$. In sum, one does not double one's chances of getting at least 1 car by switching doors. But the probability of not getting one has been reduced to as little as $1 / 15$.

\section{Conclusions}

Walking in the footsteps of Boole has made it possible, I believe, to construct a map of the mathematical structure of the Monty Hall problem in its context and of certain extensions or generalizations thereof, even if the analysis of even higher permutations remains desirable. Two components, one digital-mathematical or non-quantitative and the other quantitative, complement one another to make up this map. But the focus of the present paper is not only on this mathematical structure but also on its 
relation to the presumed digital nature of cognition as expressed in rational thought and language.

\section{Acknowledgements}

I thank Dr. Michael R. Powers, Professor of Risk Management and Insurance at Temple University's Fox School of Business and Distinguished Visiting Professor of Finance at Tshinghua University's School of Economics and Management, for reading and commenting on the present paper. In explaining the Monty Hall problem to students, Professor Powers prefers to have recourse to the method known as conditional probability. But he also believes that different approaches may supplement one another.

I am grateful to Prof. Dr. Andreas Manz, Head of Research at the Korean Institute of Science and Technology (KIST) at the University of Saarbrücken, Germany, for inviting me to participate in a workshop held at KIST on June 30-July 2, 2010 (see www.humandocument.org). At this workshop, I read a lecture entitled "How the Biological Brain Reasons: The Four Digital Operations Underlying All Rational Language and Thought.” The lecture concerned the digital analysis of rational thought and language. The ideas presented therein have inspired the present paper on the Monty Hall problem.

I was also grateful for the opportunity to be able to present some ideas on the digitalization of the analysis of rational thought and language on April 28, 2011 in a lecture entitled "The Inevitable Digitization of Language Analysis" and read in the Department of Near \& Middle Eastern Civilizations of the University of Toronto, in the framework of an Information and Discussion Session on the topic "Does It All Add Up? Quantitative Reasoning (QR) in the Humanities."

Finally, I thank two anonymous reviewers of an earlier version of this paper for their critical and penetrating comments. These comments have necessitated a complete overhaul of the paper.

\section{References}

[1] J. Rosenhouse, "The Monty Hall Problem: The Remarkable Story Behind Math's Most Contentious Brainteaser,” Oxford University Press, New York and Oxford, 2009.

[2] J. Gill, "Bayesian Methods," International Encyclopedia of Statistical Science, Springer, Berlin and New York, 2010, pp. 8-10.

[3] R. D. Gill, "The Monty Hall Problem Is not a Probability Puzzle (It's a Challenge in Mathematical Modelling)," Statistica Neerlandica, Vol. 65, No. 1, 2010, pp. 57-71. doi:10.111/j.1467-9574.2010.00474.x

[4] J. S. Rosenthal, "Monty Hall, Monty Fall, Monty Crawl," Math Horizons, Vol. 16, No. 1, September Issue, 2008, pp. 5-7.

[5] I. Grattan-Guinness, “The Search for Mathematical Roots," Princeton University Press, Princeton and Oxford, 2000.

[6] "The symbols for "and" (+) and "or" (-), and for closed-"true" (0) and open-"false" (1) as used in switching algebra, are the inverse of the conventions used in the algebra of logic. However, because of the perfect duality inherent in the algebra, the postulates and theorems are not affected" (W. Keister, A.E. Ritchie, S.H. Washburn, "The Design of Switching Circuits," The Bell Telephone Laboratories Series, D. Van Nostrand Company, New York, Toronto, and London, 1951, p. 70 note *).

[7] M. Helft, "Google Can Now Say No to 'Raw Fish Shoes' in 52 Languages,” The New York Times, March 9, 2010, pp. A1 and A3.

[8] L. Depuydt, "The Other Mathematics: Language and Logic in Egyptian and in General,” Gorgias Press, Piscataway, New Jersey, 2008, pp. 93-95.

[9] J. Venn, “Symbolic Logic," 2nd Edition, Macmillan and Company, London and New York, 1894, pp. 245-255.

[10] E. Schröder, "Vorlesungen über Die Algebra der Logik (Exakte Logik),” J. C. Hinrichs, Leipzig, Vol. 1, 1890, p. 319.

[11] L. Depuydt, "Zur Unausweichlichen Digitalisierung der Sprachbetrachtung: 'Allein,' 'anderer,' ‘auch,' ‘einziger,' '(seiner)seits,' und 'selbst' als digitales Wortfeld im Ägyptisch-Koptischen und im Allgemeinen” ("On the Unavoidable Digitalization of Language Analysis: 'Alone,' 'Other,' 'Also,' 'Only,' 'On (his) part,' and 'Self' as a Lexical Field of Digital Purport in Egyptian-Coptic and in General”), to appear in the series Aegyptiaca Monasteriensia as part of the acts of the Workshop "Lexical Fields, Semantics and Lexicography” held 5-7 November, 2010 at the University of Münster, Germany.

[12] L. Depuydt, "The Other Mathematics: Language and Logic in Egyptian and in General,” Piscataway, New Jersey, 2008, pp. 285-306.

[13] Th. Hailperin, "Boole's Logic and Probability,” NorthHolland Publishing Company, New York and Oxford, 1976, p. 131. Also see the 2nd edition, 1986, p. 215. 